\title{
Artifact versus arrhythmia in pseudo-polymorphic tachycardia; case report
}

This article was published in the following Dove Press journal:

Research Reports in Clinical Cardiology

24 April 2015

Number of times this article has been viewed

\section{Vaseem Ahmed \\ Anish Patel \\ Abhishek Sharma \\ Dennis Bloomfield}

Department of Medicine, Richmond University Medical Center, Staten Island, NY, USA
Correspondence: Vaseem Ahmed Department of Medicine, Richmond University Medical Center, 355 Bard Avenue, Staten Island, NY 10310 , USA $\mathrm{Tel}+\mathrm{I} 83279043$ I8

Email vahmed729@gmail.com
Abstract: We present the case of a young male patient in sinus rhythm whose electrocardiogram (ECG) was initially misinterpreted as ventricular tachycardia. Electrocardiographic artifact appearing to be ventricular tachycardia commonly occurs and ECG criteria have been described to aid in the discrimination between artifact and true arrhythmia. There are many causes of artifacts and prompt recognition is important to prevent unnecessary interventions.

Keywords: artifact, ventricular tachycardia, pseudo-ventricular tachycardia, notch sign, sinus sign

\section{Introduction}

Ventricular tachycardia (VT) has a known association with hemodynamic collapse and a risk of sudden cardiac death. For this reason, its electrocardiographic presence in a patient should be evaluated and managed rapidly. Artifact on electrocardiograms (ECGs) however, can be misinterpreted as VT and this possibility should be considered in an otherwise asymptomatic patient to avoid unnecessary diagnostic or therapeutic interventions. We present the case of a 48-year-old male whose telemetry rhythm strips appeared to be polymorphic VT but were subsequently confirmed to be artifact.

\section{Case report}

A 48-year-old male with no known medical history was transferred to a telemetry unit from intensive care after resolution of meningitis complicated by septic shock. Baseline ECG at the time of admission showed normal sinus rhythm and the patient denied any history of structural heart disease or dysrhythmia (Figure 1). During his hospitalization the medical team was notified by a telemetry technician after noticing sustained polymorphic VT on the monitor. Blood pressure at the time of the apparent arrhythmia was $90 / 64 \mathrm{mmHg}$, pulse was present and the patient was clinically asymptomatic. The rhythm on the telemetry monitor was re-examined and expanded to include all ECG leads and it was then noted that normal sinus rhythm was present in two of the seven leads (Figure 2). This finding was most suggestive of pseudo-VT, an incidental artifact requiring no further management or investigation.

\section{Discussion}

Unlike ischemic changes which are present in specific ECG leads depending on the site of an anatomic lesion, ventricular arrhythmias must be evident in all ECG leads. Given that our patient had normal sinus rhythm in two leads, VT could be safely excluded from 


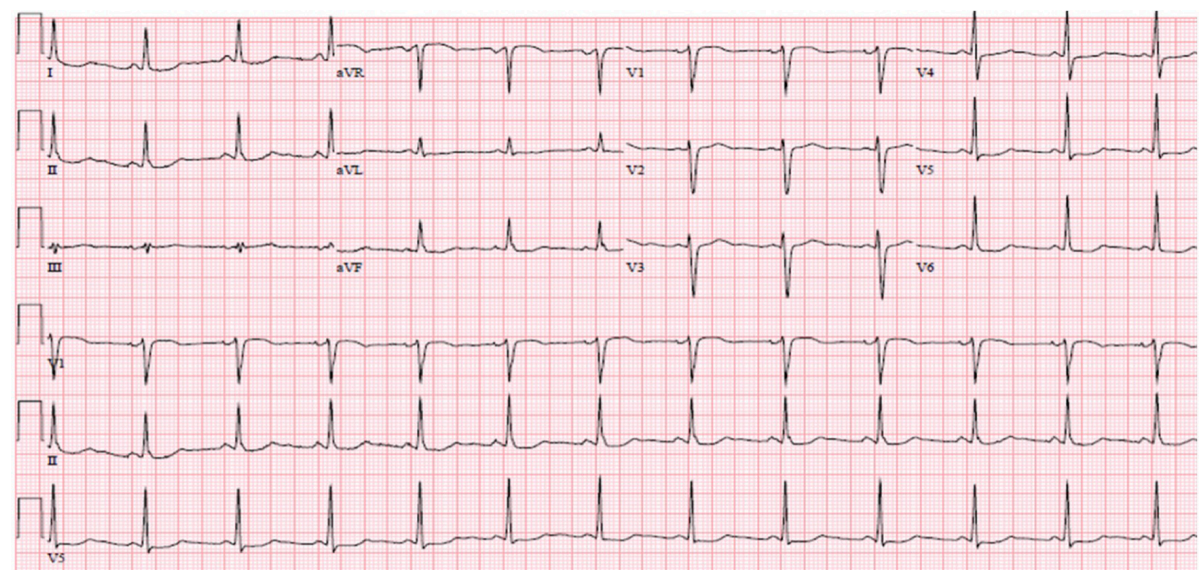

Figure I Resting electrocardiogram on admission showing normal sinus rhythm, heart rate approximately 75 beats per minute.

the differential diagnosis of the patient's unusual findings. While it can sometimes be a challenge to correctly identify a patient's rhythm, Huang et al have proposed a tested ECG algorithm to differentiate tremor induced pseudo-VT and true VT. ${ }^{1}$ According to the algorithm, the presence of any of the three characteristic signs will exclude true VT as the diagnosis. Our patient's tracing has the characteristic "sinus sign", wherein sinus rhythm is observed in a frontal or limb lead as demonstrated in leads III and V1 (Figure 2). The explanation is that if the upper limbs are not affected by

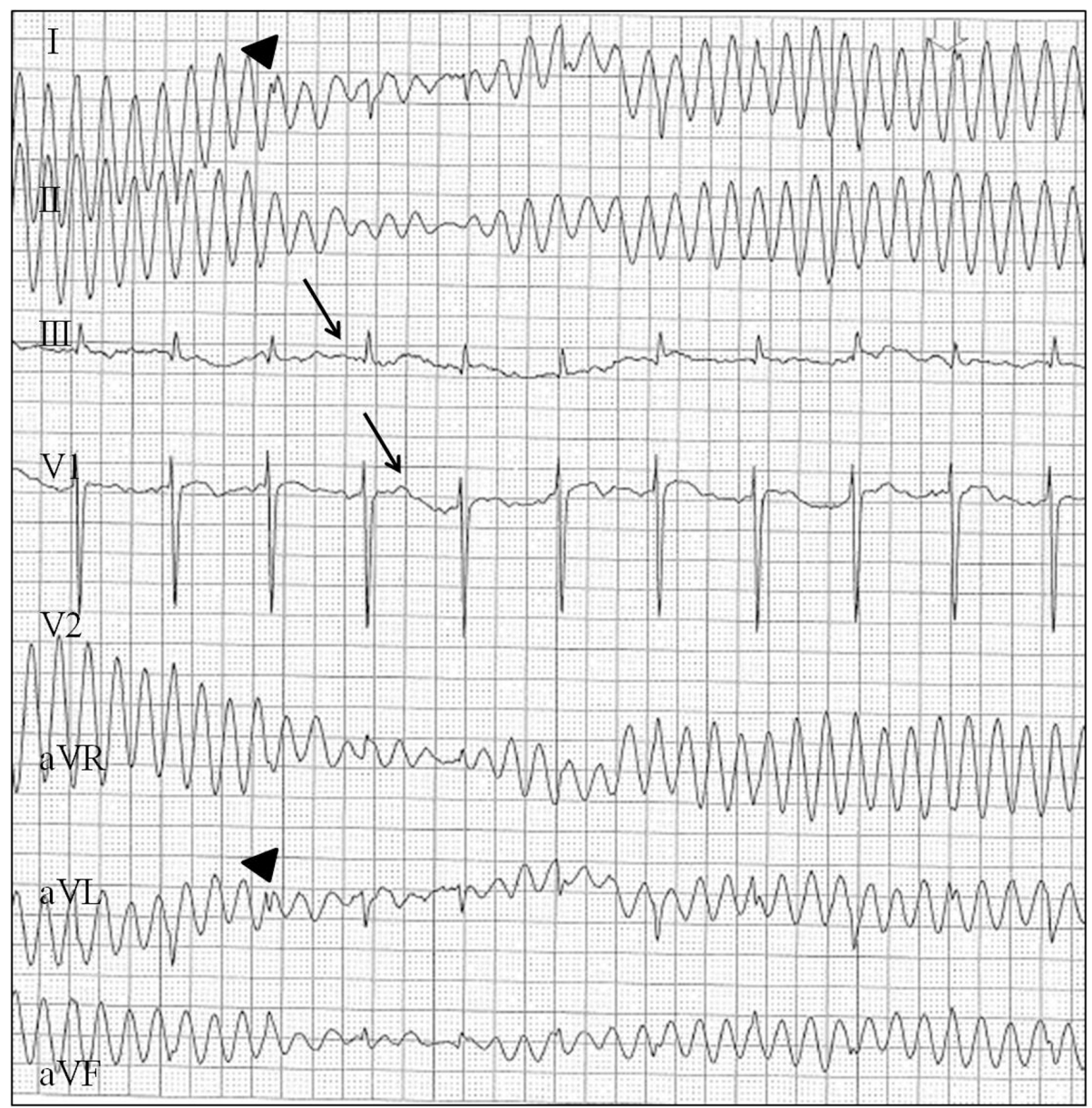

Figure 2 Expanded telemetry strips to include all electrocardiogram leads.

Notes: Note normal sinus rhythm in leads III and VI (arrow heads), characteristic of the "sinus sign". Large arrows indicate the "notch sign". 
tremor, sinus rhythm will be seen in the corresponding leads. The "notch" sign, also present in our patient is seen where a notch is present on a QRS complex and represents the length of the sinus cycle (Figure 2). The final sign described in the algorithm is the "spike sign" which are small spikes found within QRS complexes. According to the algorithm, the absence of all three signs will lead to the diagnosis of true VT. This algorithm was tested in a hospital and was able to correctly diagnose $97.3 \%$ of cases. The application of Huang's algorithm to our patient's telemetry strips is one clinical tool that can be used to differentiate arrhythmia from artifact in the case of questionable electrocardiographic features.

In the absence of clinical correlation, our patient's rhythm strip may present a challenge because of how closely it mimics VT. The wide QRS complexes suggest VT and the twisting nature of the QRS complexes around an isoelectric line may be interpreted as polymorphic. Possible explanations for the pseudo-VT seen in this case include tremor, patient movement or disruption in lead placement, all common scenarios in the inpatient setting. Knight et al examined patients who were misdiagnosed with VT and consequently underwent unnecessary interventions ranging from diagnostic coronary angiogram to permanent pacemaker or defibrillator implantation in addition to medical therapy. ${ }^{2}$ They also found that misdiagnosis occurred by cardiologists, emergency medicine physicians, and house officers, highlighting the importance of recognition of artifact and true arrhythmia across all specialties. ${ }^{3}$ The investigators followed up these findings with a survey of internists, cardiologists, and electrophysiologists who were shown an ECG tracing of artifact and asked to make an interpretation. Strikingly, $94 \%$ of internists, $58 \%$ of cardiologists, and $38 \%$ of electrophysiologists misdiagnosed the ECG as VT. Of these, $53 \%$ of cardiologists and $88 \%$ of electrophysiologists recommended further invasive management or treatment. The misdiagnosis of artifact is likely more common than it is reported and has both economic and medical implications.

Tremor is the most common cause of electrocardiographic artifact and Parkinsonian tremor mimicking VT leading to unnecessary intervention has been reported in the literature. ${ }^{4,5}$ Other etiologies of tremor as well as patient agitation may cause interference on a telemetry monitor altering the rhythm displayed. ECG lead misplacement is another common issue faced in both the inpatient and outpatient settings. ${ }^{6}$ This is in part due to the variations in lead placement, time constraints in emergencies, and lack of operator experience. ${ }^{7,8}$ The increasing use of mobile phones particularly in the hospital can also create ECG interference and mimic VT. ${ }^{9}$ Rapid physical examination and identification of any potential causes of artifact should be part of the initial bedside evaluation.

\section{Conclusion}

ECG findings of a patient are part of a broader clinical picture and should not be viewed in isolation. They also demonstrate the utility of the ECG algorithm developed by Huang et al which can be used to differentiate pseudo-VT from true VT in an uncertain circumstance. Our case further highlights the importance of examining all ECG leads for discrepancies in rhythm before subjecting a patient to potentially harmful and unnecessary interventions. It is of utmost importance to clinically assess a patient for symptoms suggestive of hemodynamic instability. The use of these considerations can result in fewer unnecessary interventions for a patient and less distress to the medical team.

\section{Disclosure}

The authors have no disclosures or conflicts of interest to report.

\section{References}

1. Huang CY, Shan DE, Lai CH, et al. An accurate electrocardiographic algorithm for differentiation of tremor-induced pseudo-ventricular tachycardia and true ventricular tachycardia. Int J Cardiol. 2006;111(1): 163-165.

2. Knight BP, Pelosi F, Michaud GF, Strickberger SA, Morady F. Clinical consequences of electrocardiographic artifact mimicking ventricular tachycardia. N Engl J Med. 1999;341(17):1270-1274.

3. Knight BP, Pelosi F, Michaud GF, Strickberger SA, Morady F. Physician interpretation of electrocardiographic artifact that mimics ventricular tachycardia. Am J Med. 2001;110(5):335-338.

4. Bhatia L, Turner, DR. Parkinson's tremor mimicking ventricular tachycardia. Age Ageing. 2005;34(4):410-411.

5. Krasnow AZ, Bloomfield DK. Artifacts in portable electrocardiographic monitoring. Am Heart J. 1976;91(3):349-357.

6. Rudiger A, Hellermann JP, Mukherjeec R, Follath F, Turia J. Electrocardiographic artifacts due to electrode misplacement and their frequency in different clinical settings. Am J Emerg Med. 2007;25(2): 174-178.

7. Bond RR, Finlay DD, Nugent CD. The effects of electrode misplacement on clinicians' interpretation of the standard 12-lead electrocardiogram. Eur J Intern Med. 2012;23(7):610-615

8. Harrigan RA, Chan TC, Brady WJ. Electrocardiographic electrode misplacement, misconnection, artifact. J Emerg Med. 2012;43(6): 1038-1044.

9. Duan X. Electrocardiographic artifact due to a mobile phone mimicking ventricular tachycardia. J Electrocardiol. 2014;47(3):333-334. 
Research Reports in Clinical Cardiology

Dovepress

\section{Publish your work in this journal}

Research Reports in Clinical Cardiology is an international, peerreviewed, open access journal publishing original research, reports, editorials, reviews and commentaries on all areas of cardiology in the clinic and laboratory. The manuscript management system is completely online and includes a very quick and fair peer-review system.

Visit http://www.dovepress.com/testimonials.php to read real quotes from published authors.

Submit your manuscript here: http://www.dovepress.com/research-reports-in-clinical-cardiology-journal 